\title{
Effect of strain rate on tensile properties of sheep disc anulus fibrosus
}

\author{
M. Kasra ${ }^{\mathrm{a}}$, M. Parnianpour ${ }^{\mathrm{b}}$, A. Shirazi-Adl ${ }^{\mathrm{c}, *}$, J.L. Wang $^{\mathrm{d}}$ and M.D. Grynpas ${ }^{\mathrm{e}}$ \\ ${ }^{a}$ Department of Mechanical, Aerospace and Biomedical Engineering, The University of Tennessee, \\ Knoxville, TN, USA \\ ${ }^{\mathrm{b}}$ Department of Mechanical Engineering, Sharif University of Technology, Tehran, Iran \\ ${ }^{\mathrm{c}}$ Department of Mechanical Engineering, Ecole Polytechnique, Montreal, Quebec \\ ${ }^{\mathrm{d}}$ Institute of Biomedical Engineering, National Taiwan University, Taipei, Taiwan \\ ${ }^{\mathrm{e}}$ Department of Rehabilitation Medicine, University of Toronto and Mount Sinai Hospital, Toronto, \\ Ontario, Canada
}

Received 9 January 2004

Revised 22 April 2004

\begin{abstract}
We investigated the effect of loading rate on tensile properties of sheep bone-anulus-bone specimens in axial direction. Disc anulus Samples with adjacent bone attachments were prepared from lateral, posterior and anterior regions of sheep lumbar spinal segments. The specimens were then tested at different strain rates under non-destructive cyclic tensile loading followed by destructive tensile loading. Each specimen was prepared by embedding the bony parts in the polymethylmetacrylate (PMMA) exposing the anulus portion to support tension. The results of non-destructive cyclic tests indicated a decrease in the hysteresis energy loss as strain rate increased. In the destructive tests, no significant differences in ultimate stress, ultimate strain and strain energy density were observed at different strain rates or annulus locations. However, there was a significant increase in the modulus at linear region as strain rate increased. Two major modes of failure were observed; rupture in the anulus mid-substance and at the anulus-endplate junction. The former failure was more frequent with no clear pattern across strain rates and locations, while the latter failure at anulus-endplate junction occurred primarily at slow strain rates.
\end{abstract}

Keywords: Disc anulus, tension, strain rate, stress, failure, modulus

\section{Introduction}

A large proportion of injuries in sports and occupational activities involves loading conditions with dynamic and high strain rates. Epidemiological studies have shown that jobs requiring a higher speed of trunk motion are associated with a greater risk of industrial low back pain [21]. For soft tissues, a larger strain rate often involves higher stiffness, failure stress, and failure strain energy density whereas a lower failure strain. The rate-dependency in failure could be more pronounced in bone than in soft tissues [29]. Rupture is, therefore, expected to initiate at the soft tissue mid-substance under high strain rates whereas at the bone by avulsion at low strain rates [7,22]. The intervertebral disc similar to other

\footnotetext{
${ }^{*}$ Address for correspondence: Professor A. Shirazi-Adl, Department of Mechanical Engineering, Ecole Polytechnique, P.O. Box 6079, Station centre-ville, Montreal, Quebec, Canada H3C 3A7. Fax.: +1 514340 4176; E-mail: abshir@ meca.polymtl.ca.
} 
soft tissues is known to exhibit time dependent material properties [12,27]. Different modes of failure at higher strain rates with greater joint load and stiffness could, hence, be expected [7,27].

In order to quantify the detailed response of the spine to dynamic loads, one needs to account for the rate dependency of the intervertebral disc in tension which is an important loading condition. Disc is known to be vulnerable to prolapse under repetitive bending [3,4,11]. In repetitive bending and lifting, the risk of lumbar disc prolapse has been reported to increase by 300-600\% [19]. Bending tasks under moderate to heavy loads generate deformations in the disc anulus and large internal pressure in the nucleus pulposus that stretch the anulus fibrosus in both axial and circumferential directions, thus rendering the disc vulnerable to failure. Behaviour of the disc anulus under repeated stretching in the axial direction has a direct bearing on its susceptibility to rupture and disc prolapse. Mechanical properties of the anulus fibrosus have been investigated as a function of direction and location under shear [18] and compression [5,16,17] loads. A number of studies have co nsidered the tensile properties of the anulus fibrosus [31]. Apart from Adams and Green [2] who used bone-anulus-bone specimens and Sonoda [26] who used whole disc specimens, others have prepared boneless samples directly from the anulus either in the radial direction $[9,10,20]$, in the collagen fibre direction $[11,20,25]$ or in the circumferential direction $[1,8,9,11,30]$. Due to the reinforcing function of collagen fibrils in the tissue, the anulus samples exhibit stiffest tensile response when taken in the fibre direction and least stiff response when taken in the radial direction perpendicular to the plane of fibres. The tensile stiffness of samples in circumferential and axial directions would lie in between those in radial and fibre directions. In terms of location, the specimens at outermost layers and anterior location demonstrate highest stiffness properties whereas those at innermost layers and posterolateral location yield the lowest stiffness $[8,25]$. Dependence of tensile material properties on disc degeneration, although to a lesser degree, has also been reported $[1,10]$. The effect of strain rate on tensile properties of the anulus fibrosus was examined in the radial direction by Fujita et al. [10] reporting no significant change while varying the strain rate by as much as 10000 fold.

The current study was undertaken to identify the extent by which the tensile properties and failure mechanism of the anulus fibrosus in the axial direction at different disc locations are influenced by the loading rate. No such attempt has been reported earlier in the literature. For this purpose bone-anulusbone specimens were taken from sheep discs.

\section{Methods}

Nineteen L5-L6 motion segments were obtained from ewes aged between two and three years. Sheep discs have been shown to possess biomechanical and biochemical characteristics similar to human discs and, hence, can be used as reasonable models for human discs [6,23]. Moreover, they exhibit smaller inter-subject variability in mechanical properties compared with human discs [28]. Soft tissues and posterior elements were removed from each motion segment leaving a body-disc-body unit for each segment. Using a band saw under continuous irrigation, four vertical slices were made through anulus regions from right and left laterals, anterior (ventral) and posterior (dorsal) regions of each body-discbody unit (Fig. 1). A total of 47 specimens were chosen for the tests, each including the bones from the adjacent upper and lower vertebrae and a continuous slice of anulus fibrosus in between (Fig. 1). The specimens from anterior $(n=14)$, lateral $(n=22)$ and posterior $(n=11)$ regions were distributed randomly into three groups and tested all at three different strain rates slow, medium, and fast. The lateral and posterior specimens had a thickness of $\sim 5 \mathrm{~mm}$ and a width of $\sim 15 \mathrm{~mm}$. The anterior specimens were $\sim 8 \mathrm{~mm}$ thick and $\sim 18 \mathrm{~mm}$ wide. The anulus height was measured before each test under $20 \mathrm{~N}$ 


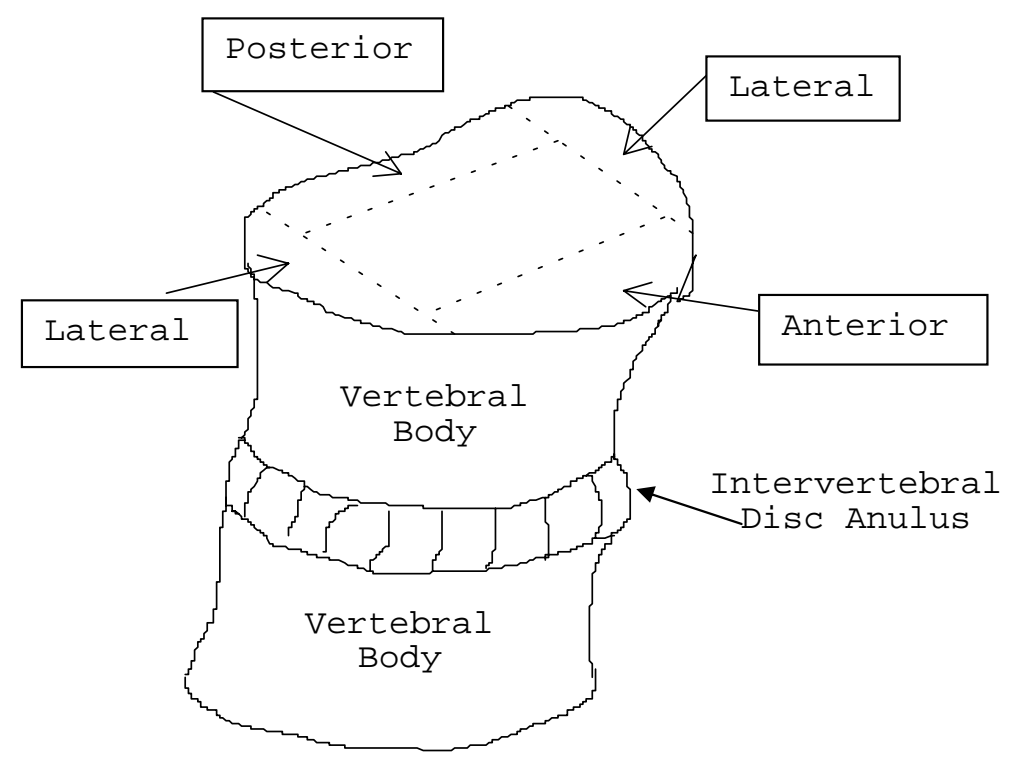

Fig. 1. Location of specimens taken from lumbar body-disc-body units.

tensile preload (baseline) using a digital calliper. Considering the variation in the height of specimens (3.0-7.1 mm), the ranges of stain rates were $0.07-0.17 \% \mathrm{~s}^{-1}$ (slow), $1.4-3.3 \% \mathrm{~s}^{-1}$ (medium), and 140$333 \% \mathrm{~s}^{-1}$ (fast). Before each test, the superior (cranial) and inferior (caudal) bones with the anulus in between, were positioned in two aluminium cups and fixed with three set screws in each cup (Fig. 2). The embedded bones were further reinforced by filling the cups with the polymethylmethacrylate (PMMA). The specimens were kept moist by spraying saline solution during the preparation and testing procedures.

Each specimen was first tested under non-destructive cyclic ramps (triangular wave) with $0.8 \mathrm{~mm}$ peak-to-peak amplitude producing $\sim 11-27 \%$ peak strain in tension, using a servo-hydraulic Instron (model 8500) mechanical testing system (Fig. 2). The loading started by 5 cycles of pre-conditioning at medium rate followed consecutively by 5 cycles at slow rate $\left(0.00313 \mathrm{~Hz}, 0.07-0.17 \% \mathrm{~s}^{-1}\right), 5$ cycles at medium rate $\left(0.0625 \mathrm{~Hz}, 1.4-3.3 \% \mathrm{~s}^{-1}\right)$ and 5 cycles at fast rate $\left(6.25 \mathrm{~Hz}, 140-333 \% \mathrm{~s}^{-1}\right)$. The results were extracted from the data recorded at the 4th cycle of each strain rate as the response was verified to stabilize after the 3 rd cycle. After the cyclic tests, the specimens from each region were tested to failure randomly at the same strain rates of slow, medium and fast. Subsequently, cross-sectional areas of anulus slices at junctions with the superior and inferior bones were also measured using computer imaging. The measured height and cross-sectional areas were used to normalize the recorded load-elongation data into stress-strain data. In the non-destructive cyclic tests, hysteresis energy loss (HE, defined as the energy loss in loading-unloading cycle normalized by the energy in loading) and modulus at the toe region $\left(\mathrm{E}_{T o e}\right)$ of the specimens were evaluated as dependent variables. In the destructive tests, failure stress, failure strain, modulus at linear region, and failure strain energy density of the specimens were measured (Fig. 3). The linear region was identified on each curve with its slope estimated by a regression analysis.

\subsection{Statistical analyses}

Multi-factorial ANOVA was first used to test the significance of independent variables (predictors), i.e., location (Loc), strain rate (SR), and their confounding effect on the dependent variables, i.e., hysteresis 


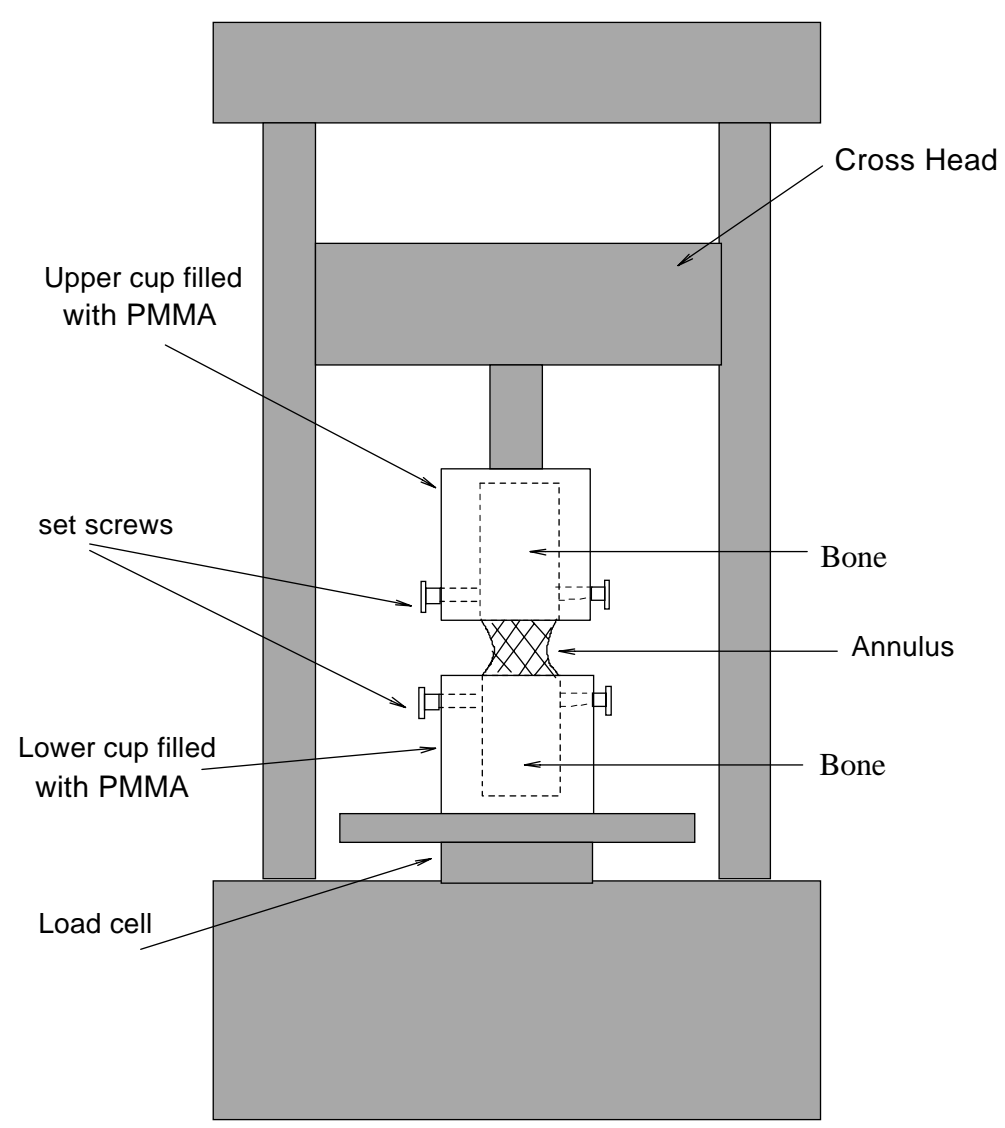

Fig. 2. Schematic of experimental setup.

(HE) and modulus at toe region $\left(\mathrm{E}_{T o e}\right)$. The significance of predictors on the dependent variables was tested. No cross subgroup effect, e.g., modulus of anterior region at slow strain rate compared with the modulus of posterior region at high strain rate, was tested at this stage. The "General Linear Model (GLM)" routine of statistical software Minitab R13 (Minitab Inc., USA) was used for this procedure. The same procedure was applied on the failure testing. After the significant predictors were found, the Post Hoc Scheffe test was conducted to delineate the difference of mechanical responses with pair wise comparisons of for example anterior site versus the lateral site. The significant level was set at $\alpha=0.05$. The statistical software SPSS R10 (SPSS Inc., USA) was used for the Post Hoc test.

\section{Results}

\subsection{Non-destructive cyclic tests}

The results of ANOVA tests indicated that mechanical properties (HE and $\mathrm{E}_{T o e}$ ) of disc anulus were significantly affected by the location and strain rate during non-destructive loading. The Post Hoc Scheffe test of the predictor strain rate and location was conducted to show the differences among levels (i.e., independent variables) for non-destructive testing (Table 1). The hysteresis energy decreased significantly from slow to medium and fast stain rates and also from posterior to lateral and anterior 
Table 1

The Post Hoc Scheffe test of mechanical responses with respect to strain rates and locations for non-destructive cyclic tests

\begin{tabular}{llclll}
\hline HE & Level & Significance $^{*}$ & $\mathrm{E}_{\text {Toe }}$ & Level & Significance \\
\hline Strain Rate & Slow-Medium & HS $(0.000)$ & Strain Rate & Slow-Medium & NS $(0.090)$ \\
& Slow-Fast & HS $(0.000)$ & & Slow-Fast & NS $(0.185)$ \\
& Medium-Fast & NS $(0.285)$ & & Medium-Fast & HS $(0.000)$ \\
Location & Anterior-Lateral & NS $(0.438)$ & Location & Anterior-Lateral & HS $(0.000)$ \\
& Anterior-Posterior & HS $(0.000)$ & & Anterior-Posterior & NS $(0.243)$ \\
& Lateral-Posterior & HS $(0.000)$ & & Lateral-Posterior & HS $(0.000)$ \\
\hline
\end{tabular}

${ }^{*} \mathrm{HS}=$ Highly Significant $(p<0.001)$, NS $=$ Not Significant $(p>0.05)$.

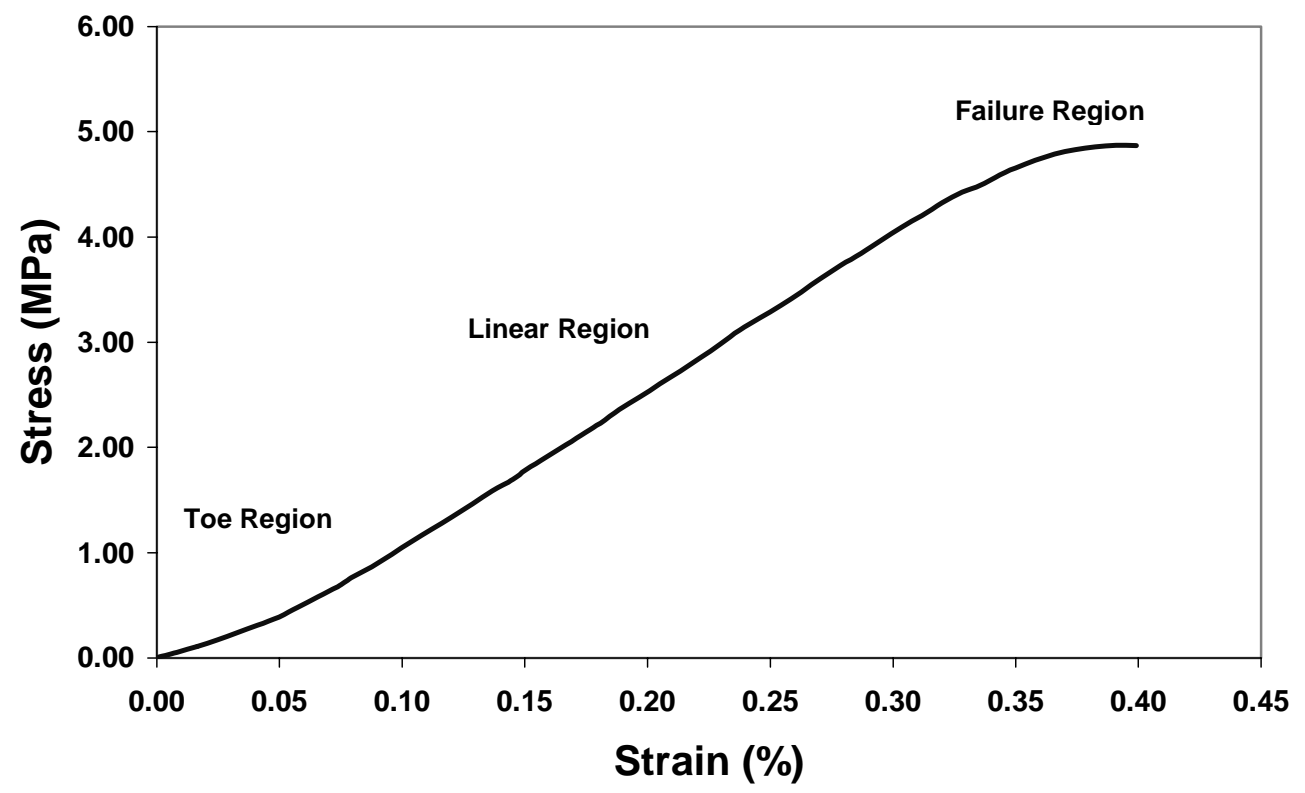

Fig. 3. A typical stress-strain curve in destructive tests showing three distinct regions; a nonlinear toe region, a linear region, and a nonlinear failure region.

regions (Fig. 4). The modulus at toe region was significantly higher at lateral side compared with anterior and posterior sides (Fig. 5).

\subsection{Destructive tests}

The multi-factorial ANOVA of mechanical responses with respect to location and strain rate, alone and combined, showed no significant changes in ultimate stress, ultimate strain, and strain energy density. This was likely due to small sample size and different modes of failure for different specimens. The ultimate stress and strain values for all locations combined were, respectively, $4.46 \pm 2.35 \mathrm{MPa}$ and 50.8 $\pm 21.1 \%$ at slow rate, $4.70 \pm 2.18 \mathrm{MPa}$ and $56.8 \pm 29.7 \%$ at medium rate, $5.28 \pm 2.84 \mathrm{MPa}$ and 40.9 $\pm 18.2 \%$ at fast rate, and finally $4.8 \pm 2.46 \mathrm{MPa}$ and $49.3 \pm 23.6 \%$ when all rates were also combined. As rates increased, a decreasing trend was observed in ultimate strain $(P=0.12)$. There was, however, a significant increase in the stiffness at linear region (Fig. 6) for the fast strain rate. Two major modes of anulus failure were observed in this study: failure in the anulus region $(n=26)$ and failure at the junction of the anulus and vertebrae at the endplates $(n=14)$. The failure location of some of the specimens 


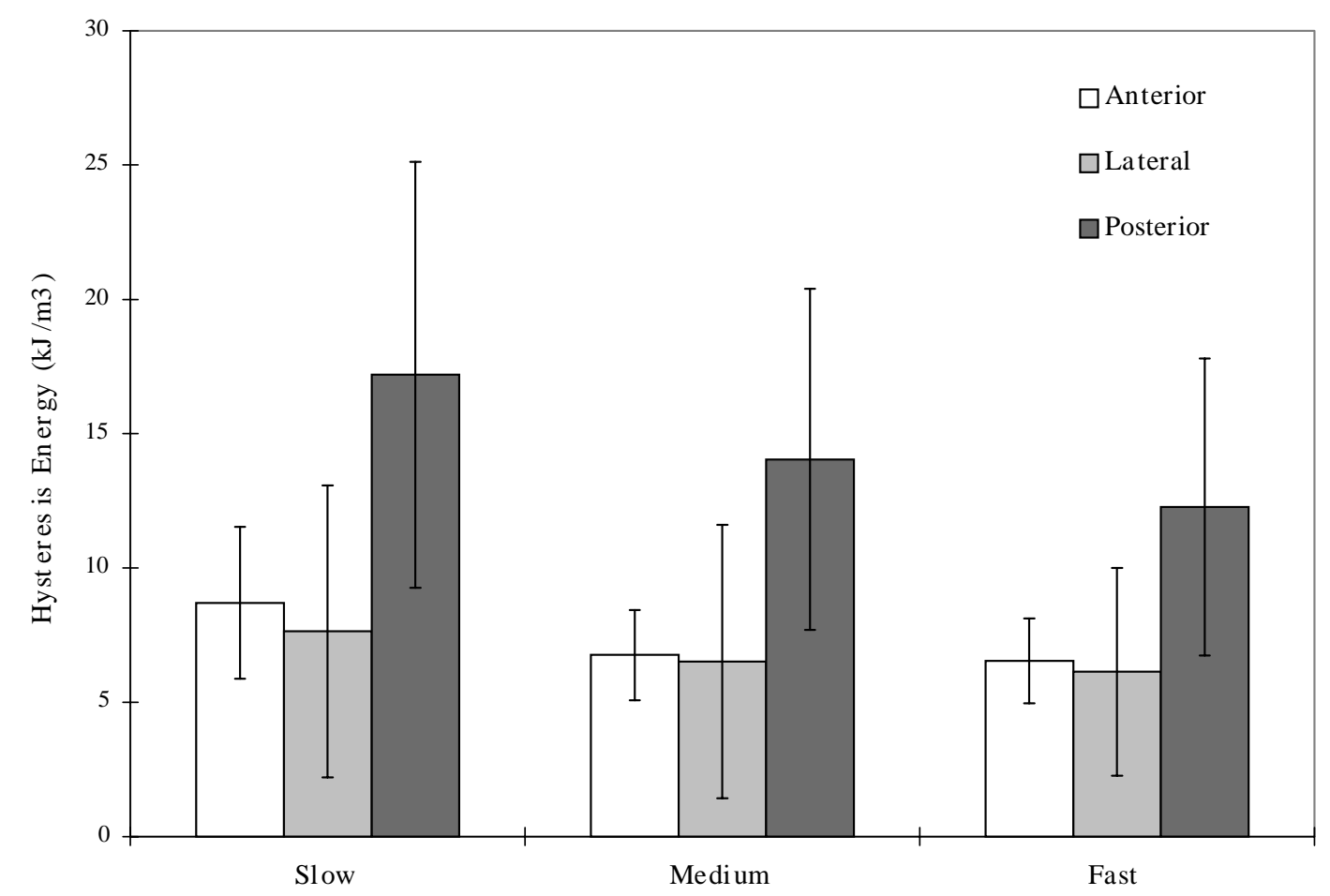

Fig. 4. Hysteresis energy (mean \pm S.D.) in non-destructive tests at different strain rates and locations.

$(n=5)$ between these two locations could not be accurately identified. Very few of the specimens failed at the bone $(n=2)$. The occurrence of the failure mode in the anulus region was spread out among different strain rates and locations. However, the failure at the anulus-endplate junction happened more frequently at the slow strain rate for the anterior and lateral specimens.

\section{Discussion}

With respect to the effect of strain rate on tensile mechanical properties, a significant increase in the modulus at linear region and a non-significant decrease in the failure strain were recorded in this study in the axial direction. Moreover, a significant decrease in hysteresis was measured in faster strain rates and in anterior and lateral locations as compared with the posterior location. Negligible effect of strain rate on mechanical properties in radial direction (i.e., cross lamellae direction) has been reported elsewhere [10], while, on the other hand, the pure collagen fibres have been found to demonstrate much greater failure stress and modulus when loaded at higher strain rates [14]. These observations suggest that the rate dependency in mechanical properties of the disc anulus as a whole could be primarily due to collagen fibres and not the annulus matrix component and, hence, should become more prevalent in tests done on samples prepared in the fibre direction. This observation could have important consequences in the temporal response and in numerical model studies of fibrous soft tissues.

Solid matrix of the disc anulus is an organized fibre reinforced structure consisting of circumferential lamellae with collagen fibres running in opposite directions from one layer to the next making angles of $\sim 30^{\circ}$ with the disc mid-plane. The collagen fibrils are the primary components of the disc in tension 


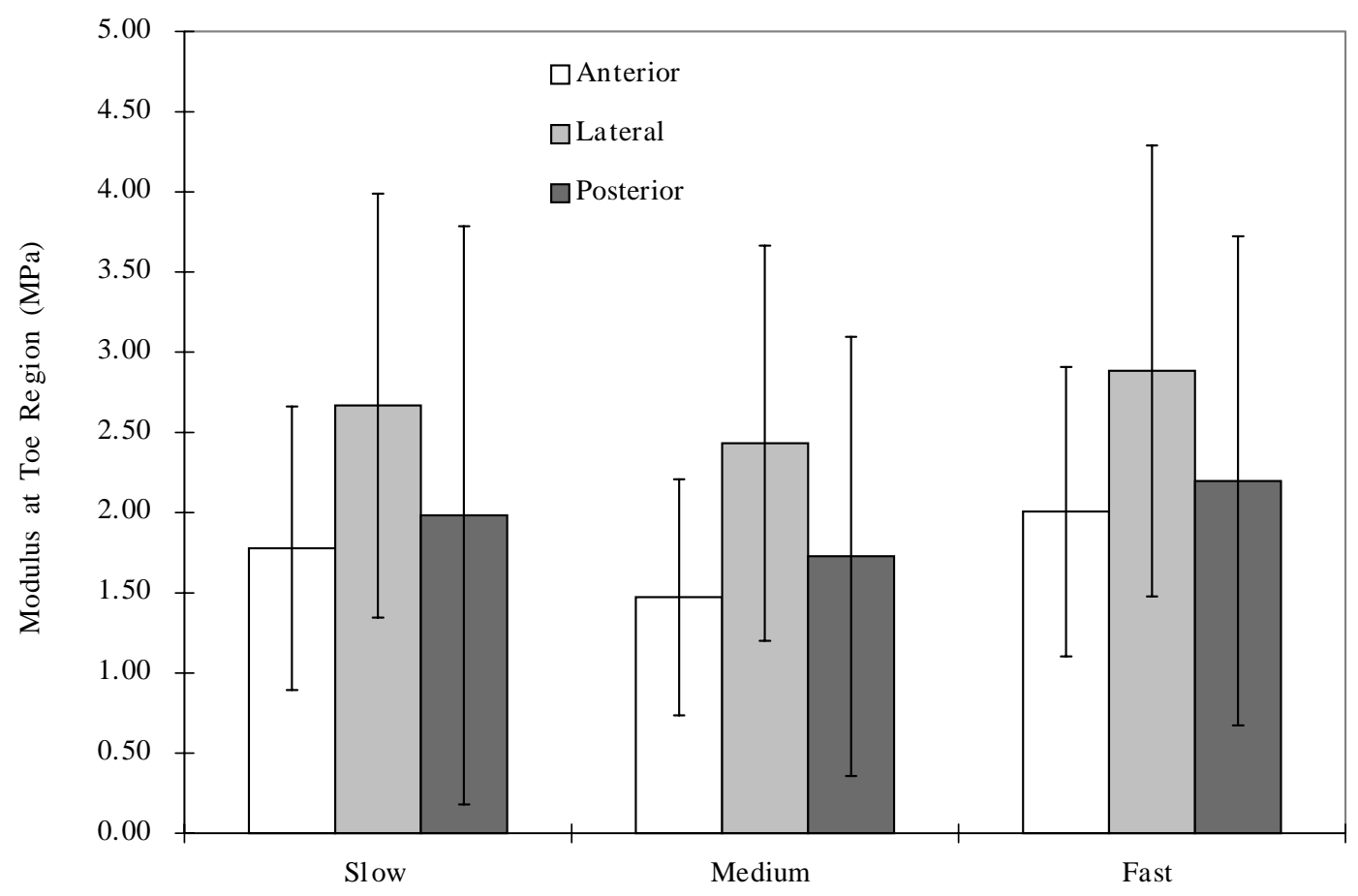

Fig. 5. Modulus (mean \pm S.D.) at toe region in non-destructive tests at different strain rates and locations.

giving rise to the tensile strength of the tissue, similar to the case in other connective tissues such as ligaments, meniscus, and cartilage,. Measurements have demonstrated substantial variations in the tensile modulus, ultimate strain and ultimate stress of the disc anulus tissue samples when taken in different directions depending on the orientation of collagen fibers. In the radial direction across the lamellae, being orthogonal to the direction of fibres, the tissue exhibits very small tensile tangent modulus and ultimate stress of $\sim 0.5 \mathrm{MPa}$ and $\sim 0.4 \mathrm{MPa}$, respectively, whereas very large ultimate strain of $144 \%$ with some variations along the radial and circumferential directions $[10,20]$. The anulus fibrosus, however, yields much stiffer response when tested in the circumferential direction especially along the collagen fibres. The modulus and ultimate stress reach $\sim 5-50 \mathrm{MPa}$ and $\sim 1-3 \mathrm{MPa}$, respectively, while the ultimate strain decreases to $\sim 10-25 \%$ in the samples taken in circumferential direction with much stiffer values at the anterior location along the outer periphery $[1,8]$. Along the fibre direction, greater tensile modulus and ultimate stress of $\sim 60-140 \mathrm{MPa}$ and $\sim 5-15 \mathrm{MPa}$, respectively have been reported [25]. Marchand and Ahmed [20] report much greater values of 210-645 MPa for the tensile modulus and 50-180 MPa for the failure tensile stress in the tangential direction which are actually reported for the collagen fibres assuming a volume fraction of $8 \%$. These latter values are in the range of those reported for tendons and collagen fibrils [13-15,24]. Our measured elastic modulus and failure stress/strain in the axial direction were in the range of those reported by others for the specimens in the circumferential direction. The ultimate stress of $4.8 \pm 2.46 \mathrm{MPa}$ measured in this study is much larger than $0.37 \pm$ $0.2 \mathrm{MPa}$ reported in the radial direction [10] but is found to be in the range of those measured along the circumferential and fibre directions [8,25]. The measured ultimate strain of $49.3 \pm 23.6 \%$ is larger than values in circumferential and fibre directions $[8,25]$ but much smaller than $144 \pm 58 \%$ reported for the radial direction [10]. Our measured moduli are found greater than those reported for the radial direction 


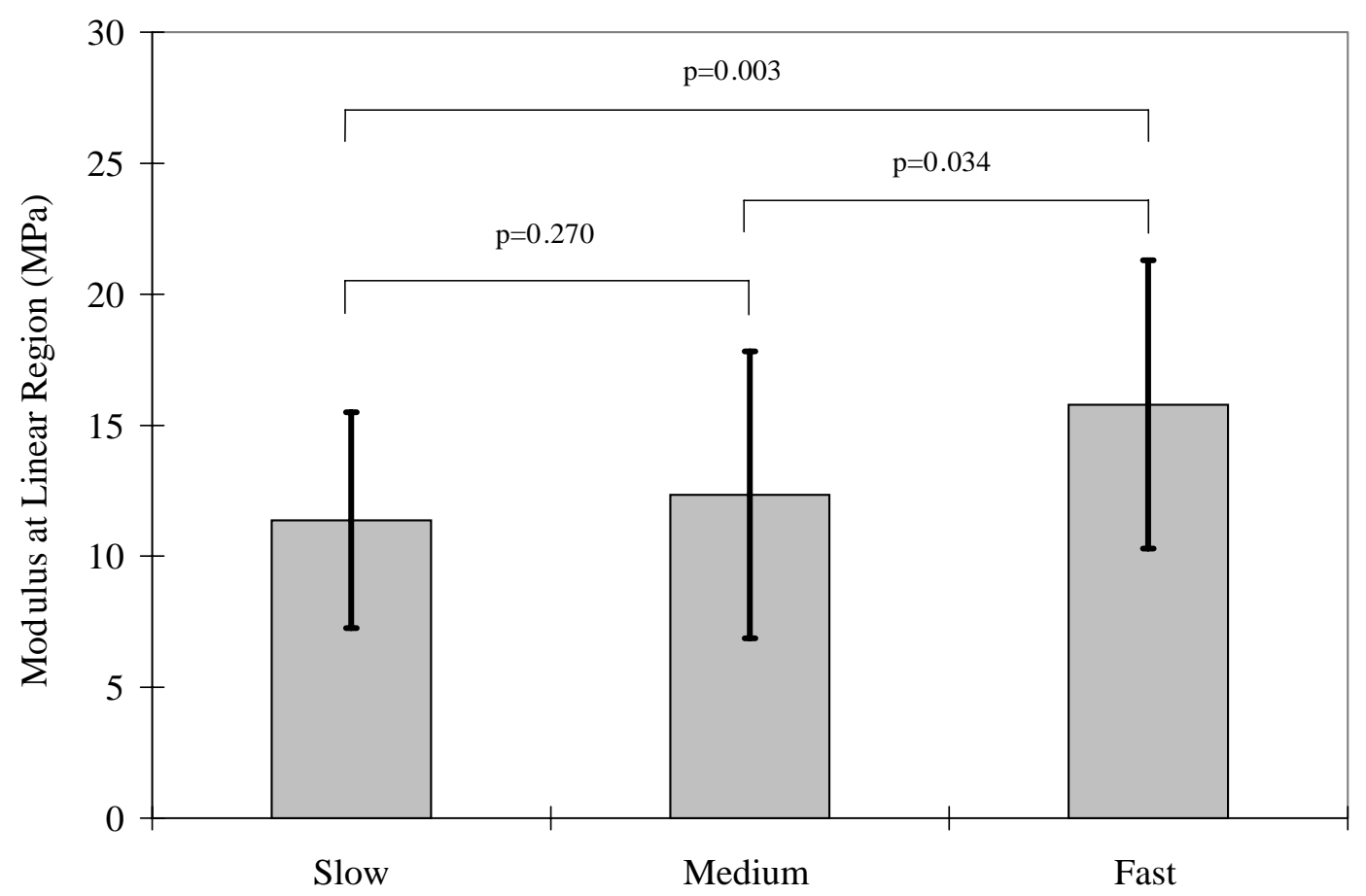

Fig. 6. Modulus (mean \pm S.D.) at linear region of stress-strain curves in destructive tests at different strain rates (data at three locations are combined). The significance level, $\mathrm{p}$, is also indicated.

but in the range of those in circumferential direction and much smaller when considering specimens in fibre direction.

The reported studies on the measurement of mechanical properties have often used regular or dumbbellshaped samples of the anulus fibrosus the preparation of which requires cutting through the collagen fibrils. The sample sizes when taken in circumferential direction are also small enough not to allow the extension of fibres from one fixated end of the specimen to the other. These disruptions in the basic structure of such reinforced composite tissue are expected to adversely influence the measurements as a whole. Adams and Green [2] demonstrated the substantial reduction in stiffness and failure load of specimens in axial tensile tests as the continuity in annulus fibres was increasingly disrupted by vertical cuts in anulus specimens. The stress and strain fields would also be influenced by the gripping method used to hold the specimens firmly in place at both ends. In the current work, to circumvent the above sources of error, the specimens were prepared in the axial direction with the disc anulus-vertebrae attachments left intact. The loads were applied and fixation was made through the bony parts at both extremities thus avoiding the tissue disruption as well as slippage and stress concentrations at end points. Due also to the relatively large width of the specimens, the continuation of fibres from one endplate to another was minimally disrupted. The large width of specimens in this study, however, could cause some inhomogeneous strain fields when the disc (or sample) height varies along the width, a situation that could present itself to some extent in specimens from the lateral regions. Moreover, since in the current work, the displacement rates were fixed rather than strain rates, there was a $<2.5$ fold differences between strain rates in each strain-rate group. These intra-group variations in strain rates, however, are much smaller and, hence, negligible when compared with the inter-group differences. 
Finally, considering the range of strain rates used in this study, our results in the axial direction indicated that the anulus fibrosus is less strain rate dependent than the bone [29]. Therefore, at higher tensile loading rates, the disc is likely more vulnerable than the bone. During material manual handling tasks while reaching a final posture required for completion of a given task, greater strain rates may place the disc at a mechanical disadvantage associated with a larger risk of injury especially in a repetitive loading environment.

\section{Acknowledgements}

This research was supported in part by a grant from the Canadian Institutes of Health Research (CIHR-Canada).

\section{References}

[1] E.R. Acaroglu, J.C. Iatridis, L.A. Setton, R.J. Foster, V.C. Mow and M. Weidenbaum, Degeneration and aging affect the tensile behavior of human lumbar anulus fibrosus, Spine 20 (1995), 2690-2701.

[2] M.A. Adams and T.P. Green, Tensile properties of the anulus fibrosus I. The contribution of fibre-matrix interactions to tensile stiffness and strength, European Spine Journal 2 (1993), 203-208.

[3] M.A. Adams and W.C. Hutton, The effect of fatigue on the lumbar intervertebral disc, J Bone Joint Surg 65B (1983), 199-203.

[4] M.A. Adams and W.C. Hutton, Has the lumbar spine a margin of safety in forward bending?, Clinical Biomechanics 1 (1986), 3-6.

[5] B.A. Best, F. Guilak, L.A. Sutton, W. Zhu, F.S. Nejad, A. Ratcliffe, M. Weidenbaum and V.C. Mow, Compressive mechanical properties of the human annulus fibrosus and their relationship to biochemical composition, Spine 19 (1994), $212-221$.

[6] J.J. Costi, T.C. Hearn and N.L. Fazzalari, The effect of hydration on the stiffness of intervertebral discs in an ovine model, Clinical Biomechanics 17 (2002), 446-455.

[7] R.D. Crowninshield and M.H. Pope, The strength and failure characteristics of rat medial collateral ligaments, The Journal of Trauma 16 (1976), 99-105.

[8] S. Ebara, J.C. Iatridis, L.A. Setton, R.J. Foster, V.C. Mow and M. Weidenbaum, Tensile properties of nondegenerate human lumbar anulus fibrosus, Spine 21 (1996), 452-461.

[9] D.M. Elliot and L.A. Sutton, Anisotropic and inhomogeneous tensile behaviour of the human annulus fibrosus: Experimental measurement and material model predictions, Journal of Biomechanical Engineering 123 (2001), 256-263.

[10] Y. Fujita, N.A. Duncan and J.C. Lotz, Radial tensile properties of the lumbar annulus fibrosus are site and degeneration dependent, Journal of Orthopaedic Research 15 (1997), 814-819.

[11] J.O. Galante, Tensile properties of the human lumbar anulus fibrosus, Acta Orthopaedica Scandinavica 100 (1967).

[12] T. Hansson, Work and back injuries, Seminars in Spine Surgery 4 (1992), 12-15.

[13] R.D. Harkness, Biological functions of collagen, Biological Review 36 (1961), 399-463.

[14] R.C. Haut, Age-dependent influence of strain rate on the tensile failure of rate-tail tenson, Journal of Biomechanical Engineering 105 (1983), 296-299.

[15] R.C. Haut, The influence of specimen length on the tensile failure properties of tendon collagen, Journal of Biomechanics 19 (1986), 951-955.

[16] G.B. Houben, M.R. Drost, J.M. Huyghe, J.D. Janssen and A.Huson, Nonhomogeneous permeability of canine annulus fibrosus 22 (1997), 7-16.

[17] J.C. Iatridis, L.A. Sutton, R.J. Foster, B.A. Rawlins, M. Weindenbaum and V.C. Mow, Degeneration effects the anisotropic and nonlinear behaviours of human annulus fibrosus in compression, Journal of Biomechanics 31 (1998), 535-544.

[18] J.C. Iatridis, S. Kumar, R.J. Foster, M. Weindenbaum and V.C. Mow, Shear mechanical properties of human annulus fibrosus, Journal of Orthopaedic Research 17 (1999), 732-737.

[19] J.L. Kelesey, P.B. Githens, A.A. White and T.R. and Holford, An epidemiologic study of lifting and twisting on the job and risk for acute prolapsed lumbar intervertebral disc, Journal of Orthopaedic Research 2 (1984), 61-66.

[20] F. Marchand and A.M. Ahmed, Mechanical properties and failure mechanisms of the lumbar disc annulus, Transaactions of the 35th Annual Meeting of The Orthopaedic Research Society, 1989, pp. 355. 
[21] W.S. Marras and K.P. Granata, A biomechanical assessment and model of axial twisting in the thoracolumbar, Spine 20 (1995), 1440-1451.

[22] F.R. Noyes, J.L. Delucas and P.J. Torvick, Biomechanics of anterior cruciate ligament failure: An analysis of strain rate sensitivity and mechanics of failure in primates, Journal of Bone \& Joint Surg 56A (1974), 236-253.

[23] J.E. Reid, J.R. Meakin, S.P. robins, J.M.S. Skakle and D.W.L. Hukins, Sheep lumbar intervertebral discs as models for human discs, Clinical Biomechanics 17 (2002), 312-314.

[24] B.J. Rigby, N. Hiral, J.D. Spikes and H. Eyring, The mechanical properties of rat tail tendon, The Journal of General Physiology 43 (1959), 265-283.

[25] D.L. Skaggs, M. Weidenbaum, J.C. Iatridis, A. Ratcliffe and V.C. Mow, Regional variation in tensile properties and biochemical composition of the human lumbar anulus fibrosus, Spine 19 (1994), 1310-1319.

[26] T. Sonoda, Studies on the strength for compression, tension and torsionh of the human vertebral column, Journal of Kyoto Prefectural Medical University 71 (1962), 659-702.

[27] J-L. Wang, M. Parnianpour, A.S. Adl and A.E. Engin, Viscoelastic finite-element analysis of a lumbar motion segment in combined compression and sagittal flexion: Effect of loading rate, Spine 25 (2000), 310-318.

[28] H.J. Wilke, A. Kettler, K.H. Wenger and L.E. Claes, Anatomy of the sheep Spine and its comparison to the human spine, The Anatomical Record 247 (1997), 542-555.

[29] T.M. Wright and W.C. Hayes, Tensile testing of bone over a wide range of strain rate, Med Biol Eng 14 (1976), 671-679.

[30] H-C. Wu and R-F. Yao, Mechanical behaviour of the human annulus fibrosus, Journal of Biomechanics 9 (1976), 1-7.

[31] H. Yamada, in: Strength of Biological Materials, F.G. Evans, eds, The Williams \& Wilkins Company, Baltimore 1970, pp. 1-105. 